\title{
Influence des paramètres matériaux sur le comportement des élastomères
}

\section{Influence of the material parameters on the mechanical properties of rubbers}

\author{
Yannick Merckel ${ }^{1}$, Mathias Brieu ${ }^{1}$ et Julie Diani ${ }^{2}$ \\ ${ }^{1}$ Laboratoire de Mécanique de Lille (UMR 8107), Ecole Centrale de Lille, Cité Scientifique, 59651 Villeneuve \\ d'Ascq, France \\ 2 Procédés et Ingénierie en Mécanique et Matériaux (UMR 8006), Arts et Métiers ParisTech, 151 Bd. de l'Hôpital, \\ 75013 Paris, France
}

\begin{abstract}
The behavior of rubber-like materials under cyclic loading induces softening, permanent stretch and anisotropy. The aim of the presented work is to characterize such damage in order to introduced relation to material properties.

Résumé. Le comportement sous sollicitations cycliques des matériaux élastomères induit un adoucissement, une déformation rémanente et une anisotropie. Le travail présenté portera sur la caractérisation de ces phénomènes d'endommagements afin d'établir des liens avec les propriétés matériaux.
\end{abstract}

\section{INTRODUCTION}

Les matériaux élastomères sont des polymères constitués de très longues chaînes macromoléculaires, réparties aléatoirement dans l'espace et réticulées, qui leur confèrent des propriétés élastiques remarquables rendant leur utilisation de plus en plus fréquente pour assumer des tâches de suspensions, tels que pour les anti vibratoires, et/ou de liaisons, comme par exemple les pneumatiques pour la liaison au sol, dans les systèmes mécaniques.

Afin de renforcer ces matériaux ou d'augmenter la résistance à la propagation de fissures, il est désormais usuel d'adjoindre à la gomme élastomère des charges de renforts (noir de carbone, silice). Cependant, l'ajout de ces charges induit au cours de chargements cycliques un adoucissement important communément nommé adoucissement Mullins (Bouasse et al. 1903 ; Mullins et al. 1947 ; Diani et al. 2009).

A ce jour, il n'y a toujours pas d'accord général sur les origines physiques conduisant à l'effet Mullins, l'influence des différents paramètres matériaux sur ce mécanisme d'endommagement ou encore les variables mécaniques susceptibles de piloter celui-ci dans des cas de chargement multi-axiaux.

Le travail présenté portera sur la caractérisation du comportement et de l'endommagement des matériaux élastomères sous chargement cyclique et la mise en évidence de l'incidence des paramètres matériaux tels que la densité de réticulation ou la fraction volumique de renforts sur le comportement et l'endommagement d'une gomme SBR. Ce travail expérimentale est un préliminaire à une modélisation, basée sur une description macromoléculaire des matériaux, qui ne sera pas présenté

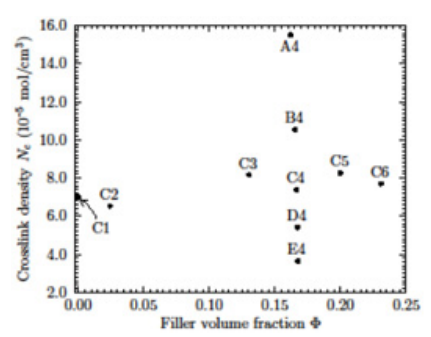

Figure 1. Plan matériaux.

dans ce résumé mais fait également l'objet de nos acticités de recherche.

\section{PLAN MATÉRIAUX}

Les matériaux élastomères sont des polymères constitués de longues chaînes macromoléculaires dont la température de transition vitreuse est inférieure aux températures d'utilisation. Ces matériaux sont ainsi utilisés dans leur domaine caoutchoutique et sont donc très grandement déformable et de très faible rigidités. Afin de diminuer leurs déformabilités et d'augmenter leurs rigidités, qui paradoxalement constitues les raisons de leurs utilisation, il est désormais classiques de créer des liaisons chimiques entre macromolécules, se caractérisant par la densité pontale de réticulation, et d'adjoindre des particules de renforts (noir de carbone, silice...).

Afin de mettre en évidence l'influence de ces paramètres matériaux sur le comportement macroscopique des élastomères renforcés, nous avons définis un plan matériau permettant d'étudier de manière différencié ces deux paramètres. Nous avons étudiés une gomme de StyrenButadienRubber (SBR) qui est un co-polymère avec 
Tableau 1. Composition des matériaux en parts par 100 (phr). Pour tous, $\mathrm{SBR}=100, \mathrm{PPD}=1,9, \mathrm{SA}=2,0, \mathrm{Zn}=2,5$.

\begin{tabular}{|l|l|l|l|l|l|l|l|l|l|l|}
\hline & A4 & B4 & C4 & D4 & E4 & C1 & C2 & C3 & C5 & C6 \\
\hline NR & 40 & 40 & 40 & 40 & 40 & 0 & 5 & 30 & 50 & 60 \\
\hline SU & 4,7 & 2,3 & 1,6 & 1,2 & 1,0 & 1,2 & 1,2 & 1,2 & 1,2 & 1,2 \\
\hline
\end{tabular}

Tableau 2. Fraction volumique (f en \%) de noir de carbone et Densité pontale $\left(\mathrm{N}_{\mathrm{c}}\right.$ en $\left.10^{-5} \mathrm{~mol} . \mathrm{cm}^{-3}\right)$.

\begin{tabular}{|l|l|l|l|l|l|l|l|l|l|l|}
\hline & A4 & B4 & C4 & D4 & E4 & C1 & C2 & C3 & C5 & C6 \\
\hline $\mathrm{f}$ & 16,2 & 16,5 & 16,6 & 16,7 & 16,7 & 0 & 2,4 & 13,0 & 20 & 23,1 \\
\hline $\mathrm{N}_{\mathrm{c}}$ & 15,5 & 10,6 & 7,4 & 5,4 & 3,6 & 7,0 & 6,5 & 8,2 & 8,3 & 7,7 \\
\hline
\end{tabular}
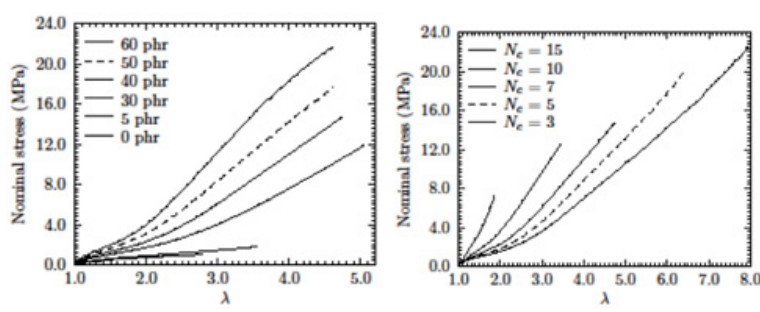

Figure 2. Réponse contrainte-déformation lors d'une traction uni axiale pour des matériaux de densité pontale equivalente $\left(\mathrm{N}_{\mathrm{c}} \sim\right.$ $7,010^{-5} \mathrm{~mol} \mathrm{~cm}^{-3}$ ), fraction volumique de renforts variable (à gauche) ou l'inverse (frac. vol. $=40 \mathrm{phr}$ ) (à droite).
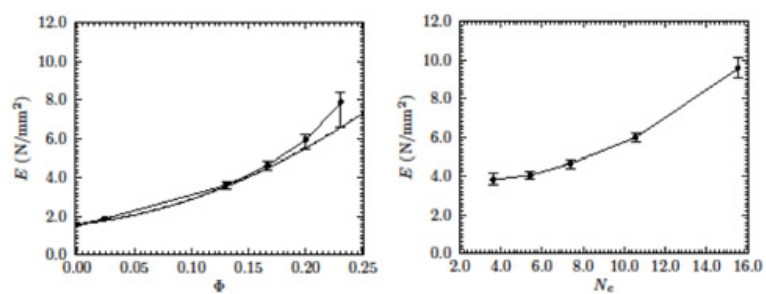

Figure 3. Dépendance du module d'Young vis-à-vis de la fraction volumique de renforts $\left(\mathrm{N}_{\mathrm{c}} \sim 7,010^{-5} \mathrm{~mol} \mathrm{~cm}^{-3}\right)$ (à gauche) ou de la densité de reticulation ( à droite).

une fraction molaire de styrène de $15 \%$. La réaction de réticulation est induite par un système de réticulation classique à base de sulfure (SU) et activé à l'aide d'un oxyde de zinc ( $\mathrm{Zn}$ ) et un acide stearique (SA) et d'une anti oxydant (PPD). La morphologie des particules de noir (NR) de carbone est caractérisée par la taille des particules élémentaire, évalué à $90 \mathrm{~m}^{2} \mathrm{~g}^{-1}$ par absorption de nitrogène absorption via une analyse de type Brunner Emmet Teller ; et par la taille des agrégats évaluée à $120 \mathrm{ml} / 100 \mathrm{~g}$ par adsorption de dibutyle-phthalate. Le plan matériaux est résumé figure 1 et détaillés tableau 1 . Les conséquences en terme de densité de réticulation et de fraction volumique de renforts sont détaillés tableau 2.

\section{COMPORTEMENT DES MATÉRIAUX}

Afin de caractériser l'influence des propriétés matériaux sur le comportement mécanique, des essais de tractions uni axiale ont été réalisés sur une machine de traction conventionnelle (INSTRON 5882). La cellule de charge utilisée est de $2 \mathrm{kN}$ et les essais ont été réalisés à vitesse de traverse constante $(10 \mathrm{~mm} / \mathrm{min})$ sur des éprouvettes
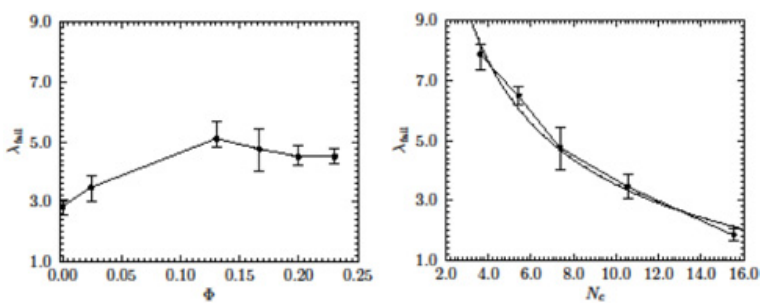

Figure 4. Effet de la fraction volumique (à gauche) ou de la densité de reticulation (à droite) sur la limite à rupture en traction uni axiale.
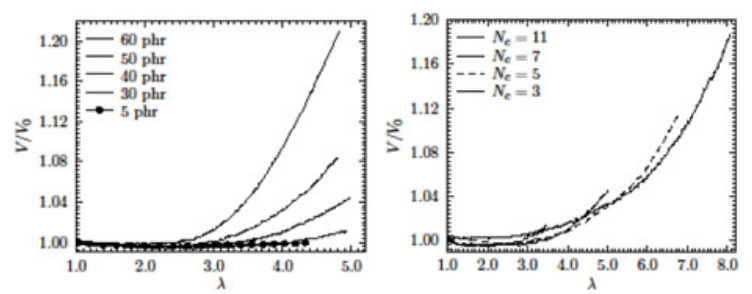

Figure 5. Variation de volume lors d'une traction uni axiale pour des densités pontales equivalente $\left(\mathrm{N}_{\mathrm{c}} \sim 7,010^{-5} \mathrm{~mol} \mathrm{~cm}^{-3}\right)$, fraction volumique de renforts variable (à gauche) ou l'inverse (40 phr) (à droite).

normalisées de $30 \mathrm{~mm}$ de longueur utile et de section utile de $10 \mathrm{~mm}^{2}$. Les déformations sont mesurées à l'aide d'un vidéo extensomètre développé en interne. Deux faces de l'éprouvette sont observées afin de mesurer les déformations dans les 3 directions de l'espace.

Une illustration des résultats expérimentaux obtenus sont présentés figures 2 et 3 .

Rajouter des particules de noir de carbone dans un élastomère permet d'augmenter la rigidité des matériaux (Khol et al., 2002) comme l'illustre la figure 2. En revanche, l'influence de la densité pontale de réticulation est nettement moins bien documentée dans la littérature.

Afin de mettre on évidence l'effet de renforcement des particules et de la densité de réticulation, nous avons déterminé le module tangent à faible déformation, ou module d'Young $\left(E=\left.\frac{d \sigma}{d \lambda}\right|_{\sigma=0}\right)$.

On peut ainsi constater que l'adjonction des particules de renforts ou l'augmentation de la densité de réticulation permettent, au même titre, de renforcer la gomme élastomère.

L'adjonction de particules de noir de carbone est également connue pour avoir une influence sur la fracture sous chargement monotone (Zhao et al., 2001) ou en 
fatigue (Auer et al., 1957). De la même manière peu de résultats sont connus concernant l'effet de la densité de réticulation. La figure 4 présente l'évolution de la limite à rupture lors d'un essai de traction monotone en fonction des caractéristiques matériaux.

Au delà de la limite à rupture, où l'on peut observer figure 4 que les deux paramètres matériaux étudiés ont une influence, nous nous sommes intéressés à la variation de volume qui dans la plupart des travaux est supposée nulle (LeCam, 2010).

La figure 5 permet de constater que la variation de volume peut se révéler, contrairement aux hypothèses présentes dans la littérature, importante. On peut constater que la variation de la densité de réticulation n'a pas d'incidence sur la variation de volume contraiment à la fraction volumique. Enfin, on peut constater, dans les deux cas que la variation de volume augmente avec la déformartion et est donc un marqueur d'un mécanisme d'endommagement.

\section{Références}

H. Bouasse, Z. Carriere. Ann. Fac. Sci. Toulouse, 1903, $3: 257-283$

L. Mullins. J. Rubber Res., 1947, 16(12):275-289

J. Diani, B. Fayolle, P. Gilormini. Eur. Polym. J., 2009, $45: 60-612$.

D. Kohls, G. Beaucage. Curr. Opin. Solid State Mat. Sci. 2002; 6, 183-194.

Zhao, J., Ghebremeskel, G.N. Rubber Chem. Technol. 2001, 74, 409-425

Auer, E.E. Doak, K.W. Schaffner, I.J. rubber world 1957, 135, 876-885.

Le Cam, J.B. Rubber Chem. Technol. 2010, 83, 247-269. 